\title{
GEOMETRIC RATIONALITY OF EQUAL-RANK SATAKE COMPACTIFICATIONS
}

\author{
LESLIE SAPER
}

Dedicated to the memory of Armand Borel, with respect and admiration

\section{Introduction}

Let $G$ be the group of real points of a semisimple algebraic group defined over $\mathbb{Q}$, let $K \subseteq G$ be a maximal compact subgroup, and let $\Gamma \subset G$ be an arithmetic subgroup. For every irreducible representation $(\sigma, U)$ of $G$, Satake [21] has constructed a compactification of the corresponding symmetric space $D=G / K$. The procedure is to pass from $(\sigma, U)$ to a representation $(\pi, V)$ having a nonzero $K$-fixed vector $v$ (a spherical representation); the compactification is then the closure of $D$ under the embedding $D \hookrightarrow \mathbb{P}(V), g K \mapsto[\pi(g) v]$. We denote the compactification by $\overline{D_{\sigma}}$ or $D_{\pi}^{*}$; it is a disjoint union of real boundary components, one of which is $D$ itself and the others are symmetric spaces of lower rank. Under certain conditions, Satake [22] creates a corresponding compactification $X_{\pi}^{*}$ of the locally symmetric space $X=\Gamma \backslash D$. These conditions were reformulated by Borel [4] and christened geometric rationality by Casselman [8].

Although the classification of Satake compactifications $D_{\pi}^{*}$ depends only on $G$ as an $\mathbb{R}$-group, geometric rationality depends a priori on the $\mathbb{Q}$-structure. Borel [4, Théorème 4.3] proved geometric rationality in the case where $(\sigma, U)$ is strongly rational ${ }^{1}$ over $\mathbb{Q}$ in the sense of [7]. Baily and Borel [3] considered the case where $D \subseteq \mathbb{C}^{N}$ is a a bounded symmetric domain and $D^{*}$ is the closure of $D$. This natural compactification is topologically equivalent to a certain Satake compactification $[21, \S 5.2],[17$, Theorem 4] and all real boundary components are Hermitian symmetric spaces. Baily and Borel prove geometric rationality, without any rationality condition on $(\sigma, U)$, by a careful consideration of root systems; another proof was given in [2]. Zucker [25] was the first to raise the

Received February 12, 2003.

2000 Mathematics Subject Classification. Primary 11F75, 22E40, 32S60, 55N33; Secondary 14G35, 22E45.

Key words and phrases. Satake compactifications, locally symmetric spaces.

This research was supported in part by the National Science Foundation through grant DMS-9870162 and through support to attend the 2002 IAS/Park City Mathematics Institute.

The original manuscript was prepared with the $\mathcal{A M}_{\mathcal{M}}$-LATEX macro system and the XY-pic package.

${ }^{1}$ This condition is stated in $[4, \S 1.6]$ but only implicitly; the term was actually coined later in [7]. Unfortunately the adverb "strongly" is sometimes omitted in the literature, e.g. see [8]. 
general question of which $(\sigma, U)$ lead to geometrically rational compactifications. In [8] Casselman gives a criterion for geometric rationality in terms of $\pi$ and the $\mathbb{Q}$-index of $G[24]$.

In this paper we establish geometric rationality in two broad situations. The first main result, Theorem 8 in $\S 5$, is that $\overline{D_{\sigma}}$ is geometrically rational if $(\sigma, U)$ is rational over $\mathbb{Q}$. This generalizes Borel's result [4] above which required that $(\sigma, U)$ be strongly rational over $\mathbb{Q}$. The second main result concerns equal-rank symmetric spaces, those that can be written as $G / K$ where $\mathbb{C}$-rank $G=\operatorname{rank} K$. Define a real equal-rank Satake compactification to be one for which each real boundary component is an equal-rank symmetric space; the possibilities have been listed by Zucker [26, (A.2)]. We prove in Theorem 20 of $\S 6$ that any real equal-rank Satake compactification is geometrically rational aside from certain $\mathbb{Q}$-rank 1 and 2 exceptions. No rationality assumption on $(\sigma, U)$ or $(\pi, V)$ is made. Every Hermitian symmetric space is an equal-rank symmetric space, so this generalizes the geometric rationality result of Baily and Borel [3]. The exceptional cases are described and treated in Theorem 22 of $\S 7$.

The equal-rank result is important since in [20] the author proved that the intersection cohomology of a real equal-rank Satake compactification $X_{\pi}^{*}$ agrees with both the intersection cohomology and the weighted cohomology of the reductive Borel-Serre compactification $\widehat{X}$. In the Hermitian case this result on intersection cohomology was conjectured independently by Rapoport [18], [19] and by Goresky and MacPherson [10], while the result on weighted cohomology in the Hermitian case was proved previously by Goresky, Harder, and MacPherson [9].

We have chosen to avoid classification theory as much as possible in order to emphasize the role played by the equal-rank condition; in particular we do not rely on Zucker's list. In fact classification theory is only used in Proposition 10 (which uses the classification of semisimple Lie algebras over $\mathbb{C}$ ), in Corollary 15 (which uses the classification of real forms of $F_{4}$ ), and in the treatment of the exceptional cases in Theorem 22.

Our main results answer several questions raised by Armand Borel [5] in a letter to the author. It is a pleasure to thank him and Bill Casselman for helpful correspondence and discussions concerning this work. Much of this work took place at the 2002 IAS/Park City Mathematics Institute on Automorphic Forms; I would like to thank the organizers of the Institute for providing a stimulating environment. I would also like to thank the referee for helpful suggestions regarding the exposition.

\section{Basic Notions}

In this paper $G$ will be the group of real points of a semisimple algebraic $\mathbb{Q}$-group, $K$ will be a maximal compact subgroup of $G$, and $D=G / K$ the associated symmetric space. For a subfield $k$ of $\mathbb{C}$ we will find it convenient to speak of a parabolic $k$-subgroup of $G$ when we strictly mean the group of real points of a parabolic $k$-subgroup of the algebraic group underlying $G$; similar 
liberties will be taken for other subgroups, such as tori or unipotent radicals, and concepts such as roots. By a representation $(\pi, V)$ of $G$ we will mean a finitedimensional $\mathbb{C}$-vector space $V$ and a homomorphism $\pi: G \rightarrow \mathrm{GL}(V)$ induced by a $\mathbb{C}$-morphism of the underlying algebraic varieties.

Fix a maximal $\mathbb{Q}$-split torus $\mathbb{Q} S$ contained in a maximal $\mathbb{R}$-split torus $\mathbb{R} S$ which itself is contained in a maximal torus $\mathbb{C} S$ of $G$ defined over $\mathbb{Q}^{2}$ Assume compatible orderings on the corresponding root systems have been chosen. For $k=\mathbb{Q}, \mathbb{R}$, or $\mathbb{C}$, let ${ }_{k} \Delta$ denote the simple $k$-roots and let ${ }_{k} W$ be the Weyl group of the $k$-root system. For $\alpha \in{ }_{k} \Delta$, let $s_{\alpha} \in{ }_{k} W$ denote the corresponding simple reflection. The Coxeter graph of the system of $k$-roots is a labeled graph with ${ }_{k} \Delta$ for its vertex set and an edge labeled $r$ between $\alpha \neq \beta$ if the product of simple reflections $s_{\alpha} s_{\beta}$ in the Weyl group has order $r>2$. If we add an arrow on an edge labeled greater than 3 which points to the shorter root we obtain the Dynkin diagram. As customary, we transfer topological terminology from the graph to ${ }_{k} \Delta$. Thus we may speak of a connected subset of ${ }_{k} \Delta$ or a path in ${ }_{k} \Delta$.

The Galois group $\operatorname{Gal}(\mathbb{C} / k)$ acts on $\mathbb{C} \Delta$ (and hence on $\mathbb{C}$-weights) via the $*$ action [7]. Namely, $g^{*}: \mathbb{C} \Delta \rightarrow \mathbb{C} \Delta$ for $g \in \operatorname{Gal}(\mathbb{C} / k)$ is defined to be $w_{g} \circ g$, where $w_{g} \in \mathbb{C} W$ is uniquely determined by $w_{g}\left(g_{\mathbb{C}} \Delta\right)={ }_{\mathbb{C}} \Delta$. In particular, if $k \subseteq \mathbb{R}$ we have $c^{*}$ where $c$ denotes complex conjugation. The opposition involution $\iota: \mathbb{C} \Delta \rightarrow \mathbb{C} \Delta$ is defined similarly by replacing $g$ with negation. The $*$-action of $\operatorname{Gal}(\mathbb{C} / k)$ and $\iota$ commute and are automorphisms of the Dynkin diagram.

Restriction of roots defines

$$
\rho_{\mathbb{C} / k}: \mathbb{C} \Delta \rightarrow{ }_{k} \Delta \cup\{0\} .
$$

The fibers $\rho_{\mathbb{C} / k}^{-1}(\alpha)$ for $\alpha \in{ }_{k} \Delta$ are nonempty Galois orbits; the fiber $\Delta_{\mathbb{C} / k}^{0}=$ $\rho_{\mathbb{C} / k}^{-1}(0)$ is Galois invariant and its elements are the $k$-anisotropic roots. For $\theta \subseteq{ }_{k} \Delta$ define $\varepsilon_{\mathbb{C} / k}(\theta)=\rho_{\mathbb{C} / k}^{-1}(\theta \cup\{0\})$. These are the types of the parabolic $k$-subgroups of $G$.

The $k$-index of $G[24]$ consists of the Dynkin diagram for $\mathbb{C} \Delta$, the subset $\Delta_{\mathbb{C} / k}^{0}$, and the $*$-action of $\operatorname{Gal}(\mathbb{C} / k)$. This can be represented diagrammatically; in the case $k=\mathbb{R}$ one recovers the Satake diagram. We will often by abuse of notation refer to the index simply as $\mathbb{C} \Delta$.

We will use the following repeatedly [7, Proposition 6.15]:

Proposition 1. For $\alpha, \beta \in{ }_{k} \Delta,\{\alpha, \beta\}$ is connected if and only if for every $\tilde{\alpha} \in \rho_{\mathbb{C} / k}^{-1}(\alpha)$, there exists $\tilde{\beta} \in \rho_{\mathbb{C} / k}^{-1}(\beta)$ and $\psi \subseteq \Delta_{\mathbb{C} / k}^{0}$ such that $\psi \cup\{\tilde{\alpha}, \tilde{\beta}\}$ is connected.

Let $\chi$ be the highest weight of an irreducible representation $(\pi, V)$ of $G$ and set $\delta=\left\{\alpha \in \mathbb{C} \Delta \mid s_{\alpha} \chi \neq \chi\right\}$. (If necessary we will denote this $\delta_{\pi}$ or $\delta_{\chi}$.) Let ${ }_{k} \chi=\left.\chi\right|_{k} S$ and define ${ }_{k} \delta \subseteq{ }_{k} \Delta$ analogously. An analogue of Proposition 1 shows that $\beta \in{ }_{k} \delta$ if and only if there exists $\tilde{\beta} \in \rho_{\mathbb{C} / k}^{-1}(\beta)$ and $\psi \subseteq \Delta_{\mathbb{C} / k}^{0}$ such

\footnotetext{
${ }^{2}$ Much of this paper would become simpler if we could assume that ${ }_{\mathbb{R}} S$ is defined over $\mathbb{Q}$. As Borel pointed out to me, this is not always possible; there are counterexamples due to Serre.
} 
that $\psi \cup\{\tilde{\beta}\}$ is connected and contains an element of $\delta$ [8, Corollary 7.2], [25, Remark in (2.4)].

For a linear combination of simple $k$-roots $\sum d_{\alpha} \alpha$, the support $\operatorname{supp}_{k}\left(\sum d_{\alpha} \alpha\right)$ is defined as $\left\{\alpha \in{ }_{k} \Delta \mid d_{\alpha} \neq 0\right\}$; the linear combination is called codominant if all $d_{\alpha} \geq 0$. A subset $\theta \subseteq{ }_{k} \Delta$ is called $\delta$-connected if every connected component of $\theta$ contains an element of ${ }_{k} \delta$. For every $k$-weight $\lambda$ of $(\pi, V)$, the difference ${ }_{k} \chi-\lambda$ is codominant with $\delta$-connected support (and integral coefficients). In fact every $\delta$-connected subset of ${ }_{k} \Delta$ arises in this way $[7,12.16]$.

For $\theta \subseteq{ }_{k} \Delta$, let $\kappa(\theta)$ denote the largest $\delta$-connected subset of $\theta$ and let $\omega(\theta)$ denote the largest subset $\Upsilon$ of ${ }_{k} \Delta$ with $\kappa(\Upsilon)=\kappa(\theta)$. Clearly

$$
\kappa(\theta) \subseteq \theta \subseteq \omega(\theta) .
$$

Let $\zeta(\theta)$ denote the complement of $\kappa(\theta)$ in $\omega(\theta)$. Equivalently, $\zeta(\theta)$ consists of those roots that are not in ${ }_{k} \delta$, not in $\kappa(\theta)$ and not joined by an edge to a root in $\kappa(\theta)$. It will sometimes be useful, especially in $\S 6$, to use the notation

$$
\theta^{+}=\theta \cup\left\{\alpha \in{ }_{k} \Delta \mid \alpha \text { is connected by an edge to a root in } \theta\right\} .
$$

We need to recall the various notions of rationality for a representation $[7$, $\S 12]$. Assume that $V$ has a $k$-structure. An irreducible representation $(\pi, V)$ is called projectively rational over $k$ if the associated projective representation $\pi^{\prime}: G \rightarrow \operatorname{PGL}(V)$ is defined over $k$. It is called rational over $k$ if $\pi: G \rightarrow \operatorname{GL}(V)$ is itself defined over $k$; it is called strongly rational over $k$ if furthermore the parabolic subgroup $P_{\pi}$ stabilizing the line spanned by the highest weight vector is defined over $k$. In terms of the highest weight $\chi$ of $(\pi, V)$, the representation is projectively rational over $k$ if and only if $\chi$ is $\operatorname{Gal}(\mathbb{C} / k)$-invariant under the $*$-action and it is strongly rational over $k$ if and only if in addition $s_{\alpha} \chi=\chi$ for all $\alpha \in \Delta_{\mathbb{C} / k}^{0}$.

An irreducible representation $(\pi, V)$ is called spherical if there exists a nonzero $K$-fixed vector $v \in V$; such a vector $v$ is unique up to scalar multiplication. By a theorem of Helgason [12], [14, Theorem 8.49], $(\pi, V)$ is spherical if and only if its highest weight $\chi$ is trivial on the maximal $\mathbb{R}$-anisotropic subtorus of $\mathbb{C} S$ and $(\chi, \alpha) /(\alpha, \alpha) \in \mathbb{Z}$ for all $\alpha \in \mathbb{R} \Delta$. In particular, a spherical representation $(\pi, V)$ is strongly rational over $\mathbb{R}$. In this case $\delta$ is $\operatorname{Gal}(\mathbb{C} / \mathbb{R})$-invariant and disjoint from $\Delta_{\mathbb{C} / \mathbb{R}}^{0} ;$ it follows that $\epsilon_{\mathbb{C} / \mathbb{R}}(\omega(\theta))=\omega\left(\epsilon_{\mathbb{C} / \mathbb{R}}(\theta)\right)$ for all $\theta \subseteq \mathbb{R} \Delta$. It also follows from [14, proof of Theorem 8.49] that a $K$-fixed vector $v$ has a nonzero component along any highest weight vector.

\section{Satake Compactifications}

As in [8] we take as our starting point an irreducible spherical representation $(\pi, V)$ of $G$, nontrivial on each noncompact $\mathbb{R}$-simple factor of $G$, with $K$-fixed vector $v$; for the relation with Satake's original construction, see $\S 3$. The Satake compactification $D_{\pi}^{*}$ associated to $(\pi, V)$ is defined to be the closure of the image of $D=G / K$ under the embedding $D \hookrightarrow \mathbb{P}(V), g K \mapsto[\pi(g) v]$. The action of $G$ on $D$ extends to an action on $D_{\pi}^{*}$. For every parabolic $\mathbb{R}$-subgroup $P$, the 
subset $D_{P, h} \subseteq D_{\pi}^{*}$ of points fixed by $N_{P}$, the unipotent radical, is called a real boundary component. The Satake compactification is the disjoint union of the real boundary components, however different $P$ may yield the same real boundary component. By associating to each real boundary component its normalizer we obtain a one-to-one correspondence.

A parabolic $\mathbb{R}$-subgroup $P$ is $\delta$-saturated if it is conjugate to a standard parabolic $\mathbb{R}$-subgroup with type $\epsilon_{\mathbb{C} / \mathbb{R}}(\omega(\theta))$ for some $\theta \subseteq \mathbb{R} \Delta$. The subgroups that arise as normalizers of real boundary components are the precisely the $\delta$ saturated parabolic $\mathbb{R}$-subgroups. The action of $P$ on $D_{P, h}$ descends to an action of its Levi quotient $L_{P}=P / N_{P}$ and the subgroup $L_{P, \ell} \subseteq L_{P}$ which fixes $D_{P, h}$ pointwise will be called the centralizer group. It has as its identity component (in the Zariski topology) the maximal normal connected $\mathbb{R}$-subgroup with simple $\mathbb{R}$ roots $\zeta(\theta)$; the simple $\mathbb{C}$-roots of $L_{P, \ell}$ are $\zeta\left(\varepsilon_{\mathbb{C} / \mathbb{R}}(\theta)\right)$. Thus $D_{P, h}$ is the symmetric space corresponding to $L_{P, h}=L_{P} / L_{P, \ell}$, a semisimple $\mathbb{R}$-group with simple $\mathbb{R}$ roots $\kappa(\theta)$; the simple $\mathbb{C}$-roots of $L_{P, h}$ are $\kappa\left(\varepsilon_{\mathbb{C} / \mathbb{R}}(\theta)\right)$, the connected components of $\varepsilon_{\mathbb{C} / \mathbb{R}}(\kappa(\theta))$ which are not wholly contained in $\Delta_{\mathbb{C} / \mathbb{R}}^{0}$.

If $D_{P, h}$ and $D_{P^{\prime}, h}$ are two standard real boundary components with normalizers having type $\epsilon_{\mathbb{C} / \mathbb{R}}(\omega(\theta))$ and $\epsilon_{\mathbb{C} / \mathbb{R}}\left(\omega\left(\theta^{\prime}\right)\right)$ respectively, define $D_{P, h} \leq D_{P^{\prime}, h}$ if $\kappa(\theta) \subseteq \kappa\left(\theta^{\prime}\right)$; this is a partial order on the standard real boundary components.

More generally the same procedure allows one to associate a Satake compactification $D_{\pi}^{*}$ to a triple $(\pi, V, v)$ consisting of a (not necessarily irreducible) representation $(\pi, V)$ which is nontrivial on each noncompact $\mathbb{R}$-simple factor of $G$ and a $K$-fixed vector $v \in V$ whose $G$-orbit spans $V$. Note that every irreducible component of $(\pi, V)$ is automatically spherical. We will only consider $(\pi, V, v)$ satisfying a very restrictive condition (although Casselman points out in [8] that the general case is worthy of further study):

(R) Assume that $(\pi, V)$ may be decomposed into the direct sum of submodules $\left(\pi_{0}, V_{0}\right)$ and $\left(\pi^{\prime}, V^{\prime}\right)$ such that $\left(\pi_{0}, V_{0}\right)$ is irreducible with highest weight $\chi_{0}$ and the difference $\chi_{0}-\chi^{\prime}$, where $\chi^{\prime}$ is the highest weight of any irreducible component of $\left(\pi^{\prime}, V^{\prime}\right)$, is codominant with $\delta_{\chi_{0}}$-connected support.

Lemma 2. If $(\pi, V, v)$ satisfies condition $(\mathrm{R})$, then $D_{\pi}^{*} \cong D_{\pi_{0}}^{*}$ as $G$-spaces.

Proof. Let $y \in D_{\pi}^{*}-D$ and let $y_{i}=\left[\pi\left(g_{i}\right) v\right] \in D$ be a sequence converging to $y$. Write $g_{i} K=k_{i} a_{i} K \in K \overline{A^{+}} K$ by the Cartan decomposition [13, IX, Theorem 1.1]; here $A$ is the identity component (in the classical topology) of $\mathbb{R}_{\mathbb{R}} S$ and $\overline{A^{+}}=\left\{a \in A \mid a^{\alpha} \geq 1, \alpha \in \mathbb{R} \Delta\right\}$. By passing to a subsequence and conjugating $\mathbb{R} S$ if necessary, we may assume that $k_{i} \rightarrow e$ in which case we may assume simply that $y_{i}=\left[\pi\left(a_{i}\right) v\right]$. Choose homogeneous coordinates $x_{\lambda}$ on $\mathbb{P}(V)$ corresponding to a basis of $\mathbb{C}$-weight vectors of $V$. We know there exists a $\mathbb{C}$-weight $\lambda$ of $V$ such that $y$ is not in the zero set of $x_{\lambda}$; let $\chi^{\prime}$ be the highest weight of an irreducible component of $V$ containing the $\mathbb{C}$-weight $\lambda$. Then $\left(x_{\chi_{0}} / x_{\lambda}\right)\left(y_{i}\right) \propto a_{i}^{\chi_{0}-\chi^{\prime}+\chi^{\prime}-\lambda} \geq 1$ since the exponent is codominant; consequently $y$ is not in the zero set of $x_{\chi_{0}}$. 
Since $D_{\pi}^{*} \cap \mathbb{P}\left(V^{\prime}\right)$ is therefore empty, the projection $V \rightarrow V / V^{\prime} \cong V_{0}$ induces a continuous map $D_{\pi}^{*} \rightarrow D_{\pi_{0}}^{*} \subseteq \mathbb{P}\left(V_{0}\right)$ of $G$-spaces. Write $v=v_{0}+v^{\prime}$. In order to see this map is bijective, we need to show that if a sequence $y_{0, i}=\left[\pi_{0}\left(a_{i}\right) v_{0}\right]$ converges to $y_{0} \in D_{\pi_{0}}^{*}$, then the sequence $y_{i}=\left[\pi\left(a_{i}\right) v\right]$ converges in $D_{\pi}^{*}$. By hypothesis, $\left(x_{\lambda_{0}} / x_{\chi_{0}}\right)\left(y_{0, i}\right) \propto a_{i}^{-\left(\chi_{0}-\lambda_{0}\right)}$ converges for every $\mathbb{C}$-weight $\lambda_{0}$ of $V_{0}$. Consequently for any $\delta_{\chi_{0}}$-connected subset $\theta_{0} \subseteq \mathbb{C} \Delta$, either $a_{i}^{-\alpha}$ converges for all $\alpha \in \theta_{0}$ or there exists $\alpha \in \theta_{0}$ with $a_{i}^{-\alpha} \rightarrow 0$; the proof is by induction on $\# \theta_{0}$. Since $a_{i}^{-\alpha} \leq 1$ for all $\alpha$, this implies

$$
a_{i}^{-\nu} \text { converges for all } \nu \text { codominant with } \delta_{\chi_{0}} \text {-connected support. }
$$

We need to show for any $\mathbb{C}$-weight $\lambda$ of an irreducible component with highest weight $\chi^{\prime}$ that $\left(x_{\lambda} / x_{\chi_{0}}\right)\left(y_{i}\right) \propto a_{i}^{-\left(\chi_{0}-\chi^{\prime}+\chi^{\prime}-\lambda\right)}$ converges. Since $\operatorname{supp}\left(\chi^{\prime}-\lambda\right)$ is $\delta_{\chi^{\prime}}$-connected and $\delta_{\chi^{\prime}} \subseteq \delta_{\chi_{0}} \cup \operatorname{supp}\left(\chi_{0}-\chi^{\prime}\right)$, it follows from $(\mathrm{R})$ that $\operatorname{supp}\left(\chi_{0}-\right.$ $\left.\chi^{\prime}\right) \cup \operatorname{supp}\left(\chi^{\prime}-\lambda\right)$ is $\delta_{\chi_{0}}$-connected. Convergence then follows from (1).

\section{Satake's Original Construction}

In this section we relate Satake's original construction [21] of compactifications of $D$ to those considered in $\S 2$. Let $(\sigma, U)$ be an irreducible representation of $G$ (not necessarily spherical) which is nontrivial on each $\mathbb{R}$-simple factor. Fix a positive definite Hermitian inner product on $U$ which is admissible in the sense that $\sigma(g)^{*}=\sigma(\theta g)^{-1}$ (here $\theta$ denotes the Cartan involution associated to $K$ ); such an inner product always exists [16] and is unique up to rescaling. Let $S(U)$ denote the corresponding real vector space of self-adjoint endomorphisms of $U$. Satake defines the compactification $\overline{D_{\sigma}}$ associated to $(\sigma, U)$ to be the closure of the image of $D$ under the embedding $D \hookrightarrow \mathbb{P} S(U), g K \mapsto \sigma(g) \sigma(g)^{*}$.

Let $g \in G$ act on $S(U)$ by $T \mapsto \sigma(g) \circ T \circ \sigma(g)^{*}=\sigma(g) \circ T \circ \sigma(\theta g)^{-1}$ and extend to a representation on $S(U)_{\mathbb{C}}$, the space of all endomorphisms of $U$. This representation is $U \otimes{ }^{\theta} U^{*}$, where ${ }^{\theta} U^{*}$ is the twist by $\theta$ of the usual contragredient representation. The identity endomorphism $I \in U \otimes{ }^{\theta} U^{*}$ is a $K$-fixed vector and we let $(\pi, V)$ denote the smallest subrepresentation containing $I$. It is clear that $\overline{D_{\sigma}}$ is isomorphic with the Satake compactification $D_{\pi}^{*}$ associated to $(\pi, V, I)$ as in $\S 2$.

Proposition 3. The triple $(\pi, V, I)$ satisfies condition $(\mathrm{R})$ with $\chi_{0}=2 \operatorname{Re} \mu$, where $\mu$ is the highest weight of $(\sigma, U)$.

Proof. The $\mathbb{C}$-weights of the $K$-fixed vector $I$ include the highest weight $\chi^{\prime}$ of any irreducible component of $(\pi, V)$. However $I=\sum_{\lambda} u_{\lambda} \otimes u_{\lambda}^{*}$, where $u_{\lambda}$ ranges over a basis of $\mathbb{C}$-weight vectors of $U$ and $u_{\lambda}^{*}$ ranges over the corresponding dual basis. Thus $\chi^{\prime}=\lambda-\theta \lambda=2 \operatorname{Re} \lambda$, where $\lambda$ is a $\mathbb{C}$-weight of $U$. Since $\operatorname{Re} \lambda$ is an $\mathbb{R}$ weight of $U$, the difference $\operatorname{Re} \mu-\operatorname{Re} \lambda$ is $\mathbb{R}$-codominant and $\operatorname{supp}_{\mathbb{R}}(\operatorname{Re} \mu-\operatorname{Re} \lambda)$ is ${ }_{\mathbb{R}} \delta_{\mu}$-connected. If $\alpha \in \mathbb{R} \Delta$, then $\operatorname{supp}_{\mathbb{C}}(2 \alpha)$ has at most 2 connected components, each containing an element of $\rho_{\mathbb{C} / \mathbb{R}}^{-1}(\alpha)$. We see that $\operatorname{supp}_{\mathbb{C}}(2 \operatorname{Re} \mu-2 \operatorname{Re} \lambda)$ is

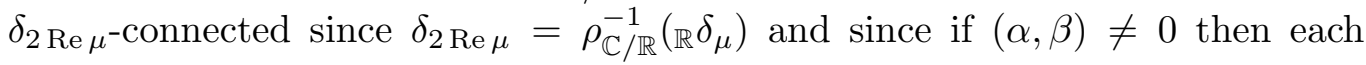


connected component of $\operatorname{supp}_{\mathbb{C}}(2 \alpha)$ is connected to a connected component of $\operatorname{supp}_{\mathbb{C}}(2 \beta)[25,(1.7)]$. Finally a component $\left(\pi_{0}, V_{0}\right)$ with highest weight $2 \operatorname{Re} \mu$ does in fact occur, since one may check that $\sum_{\left.\lambda\right|_{\mathbb{R}^{S}}=\left.\mu\right|_{\mathbb{R} S}} u_{\lambda} \otimes u_{\lambda}^{*}$ is a highest weight vector. ${ }^{3}$

Corollary 4. If $(\sigma, U)$ has highest weight $\mu$ and $\left(\pi_{0}, V_{0}\right)$ is the irreducible spherical representation with highest weight $2 \operatorname{Re} \mu$, then $\overline{D_{\mu}} \cong D_{\pi_{0}}^{*}$ as $G$-spaces.

\section{Geometric Rationality}

A real boundary component $D_{P, h}$ of $D_{\pi}^{*}$ is called rational $[3, \S \S 3.5,3.6]$ if

(i) its normalizer $P$ is defined over $\mathbb{Q}$, and

(ii) the centralizer group $L_{P, \ell}$ contains a normal subgroup $\tilde{L}_{P, \ell}$ of $L_{P}$ defined over $\mathbb{Q}$ such that $L_{P, \ell} / \tilde{L}_{R, \ell}$ is compact.

A Satake compactification $D_{\pi}^{*}$ is called geometrically rational if every real boundary component $D_{P, h}$ whose normalizer has type $\epsilon_{\mathbb{C} / \mathbb{R}}\left(\omega\left(\epsilon_{\mathbb{R} / \mathbb{Q}}(\Upsilon)\right)\right)$ for some $\delta$ connected subset $\Upsilon \subseteq \mathbb{Q} \Delta$ is rational. If $\mathbb{Q}$-rank $G=0$ (and hence $X=\Gamma \backslash D$ is already compact) any Satake compactification $D_{\pi}^{*}$ is geometrically rational, so our main interest is when $\mathbb{Q}$-rank $G>0$.

Casselman $[8$, Theorems 8.2, 8.4] proves the following criterion:

Theorem 5. The Satake compactification associated to $(\pi, V)$ is geometrically rational if and only if

(i) $\omega\left(\Delta_{\mathbb{C} / \mathbb{Q}}^{0}\right)$ is Galois invariant, and

(ii) $\kappa\left(\Delta_{\mathbb{C} / \mathbb{Q}}^{0}\right)$ is Galois invariant modulo $\mathbb{R}$-anisotropic roots, that is, the $\operatorname{Gal}(\mathbb{C} / \mathbb{Q})$ - orbit of $\kappa\left(\Delta_{\mathbb{C} / \mathbb{Q}}^{0}\right)$ is contained in $\kappa\left(\Delta_{\mathbb{C} / \mathbb{Q}}^{0}\right) \cup \Delta_{\mathbb{C} / \mathbb{R}}^{0}$.

\section{Representations Rational over $\mathbb{Q}$}

Theorem 6. The Satake compactification associated to $(\pi, V)$ is geometrically rational if $\delta$ is Galois invariant.

Proof. We need to verify (i) and (ii) from Theorem 5. Now $\kappa\left(\Delta_{\mathbb{C} / \mathbb{Q}}^{0}\right)$ is the union of those connected components of $\Delta_{\mathbb{C} / \mathbb{Q}}^{0}$ which contain an element of $\delta$. Since both $\delta$ and $\Delta_{\mathbb{C} / \mathbb{Q}}^{0}$ are Galois invariant, clearly $\kappa\left(\Delta_{\mathbb{C} / \mathbb{Q}}^{0}\right)$ is Galois invariant. This proves (ii) (in fact $\mathbb{R}$-anisotropic roots are not needed). Now $\zeta\left(\Delta_{\mathbb{C} / \mathbb{Q}}^{0}\right)$ consists of roots not in $\delta$ and not in $\kappa\left(\Delta_{\mathbb{C} / \mathbb{Q}}^{0}\right)^{+}$, hence it is Galois invariant. Since $\omega\left(\Delta_{\mathbb{C} / \mathbb{Q}}^{0}\right)=\kappa\left(\Delta_{\mathbb{C} / \mathbb{Q}}^{0}\right) \cup \zeta\left(\Delta_{\mathbb{C} / \mathbb{Q}}^{0}\right)$ this proves $(\mathrm{i})$.

Corollary 7. The Satake compactification associated to $(\pi, V)$ is geometrically rational if $(\pi, V)$ is projectively rational over $\mathbb{Q}$.

\footnotetext{
${ }^{3} \mathrm{~A}$ more direct proof that $2 \operatorname{Re} \mu$ is the highest weight of an irreducible spherical representation is given by Harish-Chandra [11, Lemma 2].
} 
Proof. By $[7, \S 12.6]$, the associated projective representation $\pi^{\prime}: G \rightarrow \operatorname{PGL}(V)$ is defined over $\mathbb{Q}$ if and only if the highest weight of $V$ is Galois invariant; thus $\delta$ is Galois invariant.

Note that in Satake's original construction, the passage from $(\sigma, U)$ to $\left(\pi_{0}, V_{0}\right)$ does not necessarily preserve rationality. Thus Corollary 7 does not settle the question of geometric rationality if $(\sigma, U)$ is assumed to be rational over $\mathbb{Q}$. Nonetheless we can prove: ${ }^{4}$

Theorem 8. The Satake compactification associated to $(\sigma, U)$ by Satake's original construction is geometrically rational if $(\sigma, U)$ is projectively rational over $\mathbb{Q}$.

Proof. By Corollary 4, we need to verify (i) and (ii) from Theorem 5 for $\delta=$ $\delta_{2 \operatorname{Re} \mu}$ under the hypothesis that $\delta_{\mu}$ is Galois invariant. By the remarks following Proposition 1 and the fact that $\delta_{\mu}$ is $c^{*}$-invariant, we can write

$\delta=\left\{\alpha \in \mathbb{C} \Delta-\Delta_{\mathbb{C} / \mathbb{R}}^{0} \mid\right.$ there exists $\psi \subseteq \Delta_{\mathbb{C} / \mathbb{R}}^{0}$ such that $\psi \cup\{\alpha\}$ is $\delta_{\mu}$-connected $\}$.

Let $\kappa=\kappa\left(\Delta_{\mathbb{C} / \mathbb{Q}}^{0}\right)$ is the union of those connected components of $\Delta_{\mathbb{C} / \mathbb{Q}}^{0}$ which contain an element of $\delta$; similarly let $\kappa_{\mu}=\kappa_{\mu}\left(\Delta_{\mathbb{C} / \mathbb{Q}}^{0}\right)$ is the union of those connected components of $\Delta_{\mathbb{C} / \mathbb{Q}}^{0}$ which contain an element of $\delta_{\mu}$. Clearly $\kappa \subseteq \kappa_{\mu}$ while $\kappa_{\mu}-\kappa$ is the union of those connected components of $\Delta_{\mathbb{C} / \mathbb{Q}}^{0}$ which lie within $\Delta_{\mathbb{C} / \mathbb{R}}^{0}$ and contain an element of $\delta_{\mu}$. Since $\kappa_{\mu}$ is Galois invariant, (ii) is satisfied.

For (i) we calculate $\mathbb{C} \Delta-\omega\left(\Delta_{\mathbb{C} / \mathbb{Q}}^{0}\right)=(\mathbb{C} \Delta-\kappa) \cap\left(\delta \cup \kappa^{+}\right)=\left(\mathbb{C} \Delta-\kappa_{\mu}\right) \cap\left(\delta \cup \kappa_{\mu}^{+}\right)$, where the second equality holds since $\kappa_{\mu}-\kappa \subseteq \Delta_{\mathbb{C} / \mathbb{R}}^{0}$ is disjoint from $\delta \cup \kappa^{+}$and since $\left(\kappa_{\mu}^{+}-\kappa^{+}\right)-\kappa_{\mu} \subseteq \delta$. Since $\delta_{\mu} \cap\left(\mathbb{C} \Delta-\Delta_{\mathbb{C} / \mathbb{Q}}^{0}\right) \subseteq \delta \subseteq\left(\delta_{\mu} \cap\left(\mathbb{C} \Delta-\Delta_{\mathbb{C} / \mathbb{Q}}^{0}\right)\right) \cup \kappa_{\mu}^{+}$, we can thus write $\mathbb{C} \Delta-\omega\left(\Delta_{\mathbb{C} / \mathbb{Q}}^{0}\right)=\left(\mathbb{C} \Delta-\kappa_{\mu}\right) \cap\left(\left(\delta_{\mu} \cap\left(\mathbb{C} \Delta-\Delta_{\mathbb{C} / \mathbb{Q}}^{0}\right)\right) \cup \kappa_{\mu}^{+}\right)$, which is clearly Galois invariant.

\section{Equal-rank Satake Compactifications}

We begin with some generalities about the structure of root systems, here applied to the simple $\mathbb{C}$-roots $\mathbb{C} \Delta$ of $G$. Let $\iota$ be the opposition involution, that is, the negative of the longest element of the Weyl group. For a connected subset $\psi \subseteq \mathbb{C} \Delta$ which is invariant under $\iota$, let $\left.\iota\right|_{\psi}$ denote the restriction, while $\iota_{\psi}$ denotes the opposition involution of the subroot system with simple roots $\psi$. Recall

$$
\psi^{+}=\psi \cup\{\alpha \in \mathbb{C} \Delta \mid \alpha \text { is connected by an edge to a root in } \psi\}
$$

Definition 9. Let $\widetilde{\mathcal{F}}$ denote the family of nonempty connected $\iota$-invariant subsets $\psi \subseteq \mathbb{C} \Delta$ for which $\iota_{\psi}=\iota_{\psi}$. Let $\mathcal{F}$ consist of those $\psi \in \widetilde{\mathcal{F}}$ such that

(i) $\psi^{+}-\psi$ modulo $\iota$ has cardinality $\leq 1$, and

\footnotetext{
${ }^{4}$ The result of Theorem 8 is asserted in [25, (3.3), (3.4)], however in [8, §9] it is noted that [25, Prop. (3.3)(ii)] is incorrect. Also the proof of [25, Prop. (3.4)] seems to implicitly assume that $(\sigma, U)$ is strongly rational over $\mathbb{R}$.
} 
(ii) there exists $\psi^{\prime} \supseteqq \psi^{+}, \psi^{\prime} \in \widetilde{\mathcal{F}}$ satisfying (i), such that all components of $\psi^{\prime}-\psi^{+}$are in $\widetilde{\mathcal{F}}$.

Let $\widetilde{\mathcal{F}}^{*} \subseteq \widetilde{\mathcal{F}}$ exclude sets of cardinality 1 which are not components of $\mathbb{\mathbb { C }} \Delta$ and for each component $C \subseteq \mathbb{C} \Delta$, let $\widetilde{\mathcal{F}}_{C}=\{\psi \in \widetilde{\mathcal{F}} \mid \psi \subseteq C\}$; similarly define $\mathcal{F}^{*}$ and $\mathcal{F}_{C}$.

We view $\mathcal{F}$ and $\widetilde{\mathcal{F}}$ as partially ordered sets by inclusion. For any partially ordered set $\mathcal{P}$, recall that $\psi^{\prime} \in \mathcal{P}$ covers $\psi \in \mathcal{P}$ if $\psi<\psi^{\prime}$ and there does not exists $\psi^{\prime \prime} \in \mathcal{P}$ with $\psi<\psi^{\prime \prime}<\psi^{\prime}$. The Hasse diagram of the partially ordered set $\mathcal{P}$ has nodes $\mathcal{P}$ and edges the cover relations; if $\psi^{\prime}$ covers $\psi$ we draw $\psi^{\prime}$ to the right of $\psi$. (For the basic terminology of partially ordered sets, see $[23$, Chapter 3], but note that in [23] $\psi^{\prime}$ is drawn above $\psi$.)

Proposition 10. The Hasse diagrams of $\mathcal{F}_{C}$ and $\widetilde{\mathcal{F}}_{C}$ are given in Figure 1. In particular if $\psi<\psi^{\prime} \in \widetilde{\mathcal{F}}$ and $\psi \in \mathcal{F}$ then $\psi^{\prime} \in \mathcal{F}$. Also if $C$ is not type $F_{4}$, then:

(i) $\widetilde{\mathcal{F}}_{C}^{*}$ is totally ordered.

(ii) Suppose $\psi, \psi^{\prime} \in \mathcal{F}_{C}$ are incomparable. If $C$ is not type $B_{n}, C_{n}$, or $G_{2}$, then one of $\psi$ and $\psi^{\prime}$ is type $A_{1}$ and $\psi \cup \psi^{\prime}$ is disconnected. If $C$ is type $B_{n}, C_{n}$, or $G_{2}$, one of $\psi$ and $\psi^{\prime}$ is type $A_{1}$ and is covered by $C$.

Proof. Recall [24] that $\left.\iota\right|_{C}$ is the unique nontrivial involution in the cases $A_{n}$ $(n>1), D_{n}(n>4$, odd $)$, and $E_{6}$, and is trivial otherwise. If $\operatorname{rank} C>1$, let $C_{0} \subseteq C$ be defined as follows: if $\left.\iota\right|_{C}$ is nontrivial, $C_{0}$ is the unique subdiagram of type $A_{2}$ or $A_{3}$ which is nontrivially stabilized under $\left.\iota\right|_{C}$; if $\left.\iota\right|_{C}$ is trivial, $C_{0}$ is the unique subdiagram of type $B_{2}, D_{4}$, or $G_{2}$. The subdiagram $C_{0}$ "determines" $\left.\iota\right|_{C}$. In particular if $\psi \subseteq C$ is a connected $\iota$-invariant subset with $\operatorname{rank} \psi>1$, then $\left.\iota\right|_{\psi}=\iota_{\psi}$ is equivalent to $C_{0}=\psi_{0}$. Such $\psi$ are easy to enumerate. For example, if $C$ is type $B_{n}$, then $\psi$ can be any connected segment containing the double bond at one end. Together with the cardinality 1 subsets, such calculations yield $\widetilde{\mathcal{F}}_{C}$. To determine $\mathcal{F}_{C}$, one then checks which of these $\psi$ satisfy the additional conditions of Definition 9. The results are pictured in Figure 1 and the proposition follows.

Definition 11. Given a Satake compactification $D_{\pi}^{*}$, let $\mathcal{B}$ denote the family of subsets $\kappa\left(\varepsilon_{\mathbb{C} / \mathbb{R}}(\theta)\right) \subseteq \mathbb{C} \Delta$, where $\theta \subseteq \mathbb{R} \Delta$ is a nonempty connected and $\delta$ connected subset. Let $\mathcal{B}^{*} \subseteq \mathcal{B}$ exclude those subsets $\kappa\left(\varepsilon_{\mathbb{C} / \mathbb{R}}(\theta)\right)$ with cardinality 1 which are not components of $\mathbb{C}_{\mathbb{C}} \Delta$. For every component $C$ of $\mathbb{C} \Delta$, set $\mathcal{B}_{C \cup c^{*} C}=$ $\left\{\psi \in \mathcal{B} \mid \psi \subseteq C \cup c^{*} C\right\}$ and $\mathcal{B}_{C \cup c^{*} C}^{*}=\mathcal{B}_{C \cup c^{*} C} \cap \mathcal{B}^{*}$.

An element of $\mathcal{B}$ is simply the set of simple roots for the automorphism group of a nontrivial standard irreducible boundary component. The correspondence $\theta \leftrightarrow \kappa\left(\varepsilon_{\mathbb{C} / \mathbb{R}}(\theta)\right)$ above is an isomorphism of partially ordered sets for the inclusion ordering. Note that in the situation to be considered below, $C \cup c^{*} C=C$.

A semisimple algebraic $\mathbb{R}$-group is equal-rank if $\mathbb{C}$-rank $G=\operatorname{rank} K$. A symmetric space $D$ is equal-rank if $D=G / K$ for an equal-rank group $G$. A real equal-rank Satake compactification is a Satake compactification $D_{\pi}^{*}$ for which all 
(Type $A_{n}, n$ odd)

(Type $A_{n}, n$ even)

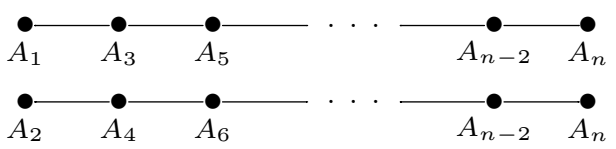

(Type $B_{n}$ )

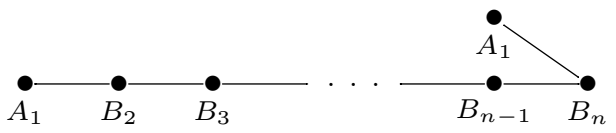

(Type $C_{n}$ )

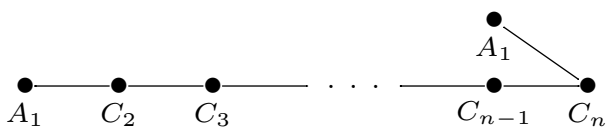

(Type $D_{n}, n$ odd)

(Type $D_{n}, n>4$ even)

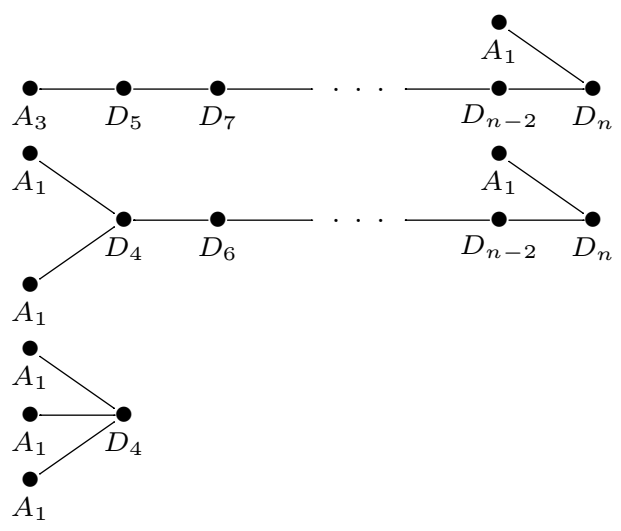

(Type $E_{6}$ )

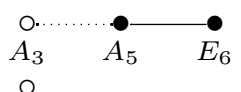

(Type $E_{7}$ )

(Type $E_{8}$ )
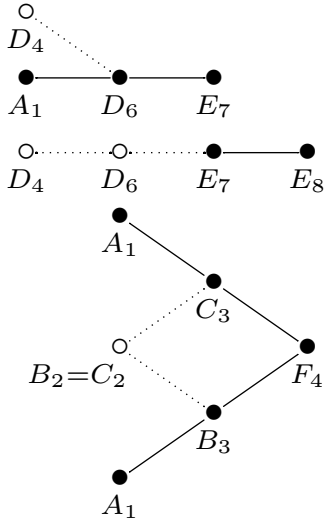

(Type $F_{4}$ )

(Type $G_{2}$ )

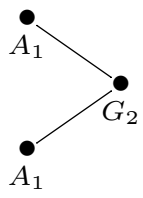

FiguRE 1. Hasse Diagrams of $\widetilde{\mathcal{F}}_{C}\left(\bullet=\right.$ node of $\mathcal{F}_{C} ; \circ=$ node of $\left.\widetilde{\mathcal{F}}_{C}-\mathcal{F}_{C}\right)$. Each node $\psi$ is labeled by the type of the root system generated by $\psi$; for simplicity, $A_{1}$ nodes in $\widetilde{\mathcal{F}}_{C}-\mathcal{F}_{C}$ are omitted. 
real boundary components $D_{P, h}$ are equal-rank. In the case of a real equal-rank Satake compactification we wish to relate $\mathcal{B}$ (which depends on the $\mathbb{R}$-structure of $G$ and the given Satake compactification) with $\mathcal{F}$ (which only depends on $G$ as a $\mathbb{C}$-group). We begin with a basic lemma.

Lemma 12 (Borel and Casselman). A semisimple $\mathbb{R}$-group $G$ is equal-rank if and only if $c^{*}=\iota$, where $c \in \operatorname{Gal}(\mathbb{C} / \mathbb{R})$ is complex conjugation.

Proof. See $[6, \S 1.2(1)$ and Corollary 1.6(b)].

Corollary 13. If a semisimple $\mathbb{Q}$-group $G$ is equal-rank, then $c^{*}$ commutes with $g^{*}$ for all $g \in \operatorname{Gal}(\mathbb{C} / \mathbb{Q})$.

Lemma 14. Let $D_{\pi}^{*}$ be a real equal-rank Satake compactification. Then $\mathcal{B} \subseteq \widetilde{\mathcal{F}}$, every component of $\Delta_{\mathbb{C} / \mathbb{R}}^{0}$ belongs to $\widetilde{\mathcal{F}}$, and every component $C$ of $\mathbb{C} \Delta$ is $c^{*}$ stable.

Proof. Let $c_{\psi}^{*}$ denote the $*$-action of $c$ for the subroot system with simple roots $\psi=\kappa\left(\varepsilon_{\mathbb{C} / \mathbb{R}}(\theta)\right)$; one may check that $c_{\psi}^{*}=\left.c^{*}\right|_{\psi}$. By our equal-rank assumption and Lemma $12, c_{\psi}^{*}=\iota_{\psi}$ and $c^{*}=\iota$. It follows that $\psi$ is $\iota$-stable and that $\left.\iota\right|_{\psi}=\iota_{\psi}$. It also follows that $\psi$ is connected, since otherwise $c_{\psi}^{*}$ would interchange the components and $\iota_{\psi}$ would preserve them. The same argument applies to a component $\psi$ of $\Delta_{\mathbb{C} / \mathbb{R}}^{0}$ since $c_{\psi}^{*}=\left.c^{*}\right|_{\psi}$ and $\psi$ is the index of an equal-rank (even $\mathbb{R}$-anisotropic) group.

Corollary 15. Let $D_{\pi}^{*}$ be a real equal-rank Satake compactification. If a component $C$ of $\mathbb{C} \Delta$ has type $F_{4}$, then $\mathbb{R}$-rank $C=0$ or 1 .

Proof. If $\mathbb{R}$-rank $C=4$, then $\mathcal{B}_{C} \subseteq \widetilde{\mathcal{F}}_{C}$ contains a chain of length 4 . By Figure 1, the chain must begin with one of the roots in the $B_{2}$ subdiagram; this root must therefore belong to $\delta$. But then $\mathcal{B}_{C}$ would also contain an element of type $A_{2}$ which is excluded by the figure. Thus $\mathbb{R}$-rank $C \leq 3$. However $F_{4}$ only has real forms with $\mathbb{R}$-rank $=0,1$, and $4[1, \S 5.9]$, [13, Chapter X, Table V].

Corollary 16. Let $D_{\pi}^{*}$ be a real equal-rank Satake compactification. If $\theta \subseteq{ }_{\mathbb{R}} C$ is a $\delta$-connected subset of a connected component ${ }_{\mathbb{R}} C$ of ${ }_{\mathbb{R}} \Delta$, then $\theta$ is connected. Consequently $\kappa\left(\varepsilon_{\mathbb{C} / \mathbb{R}}(\theta)\right) \subseteq \mathbb{C} \Delta$ is connected and (if nonempty) belongs to $\mathcal{B}$.

Proof. Let $C=\varepsilon_{\mathbb{C} / \mathbb{R}}(\mathbb{R} C)$ be the corresponding component of $\mathbb{C}_{\mathbb{C}} \Delta ;$ it is connected by Lemma 14. We will prove that any two elements of ${ }_{\mathbb{R}} \delta \cap{ }_{\mathbb{R}} C$ are connected by an edge, from which the corollary follows. To see this, assume otherwise. Then there are clearly two distinct connected and $\delta$-connected subsets of $\underset{\mathbb{T}}{\widetilde{T}} C$ with cardinality 2. This is impossible if the type of $C$ is not $F_{4}$, since $\mathcal{B}_{C}^{*} \subseteq \widetilde{\mathcal{F}}_{C}^{*}$ is totally ordered by Proposition 10(i) and Lemma 14 . Type $F_{4}$ is excluded by Corollary 15.

We introduce the following convenient notational convention: for $i \geq 1, \psi_{i}$ will always denote some element of $\mathcal{B}$ with $\mathbb{R}$-rank $\psi_{i}=i$; let $\psi_{0}=\emptyset$. The following observation will be used repeatedly. 
Lemma 17. Let $D_{\pi}^{*}$ be a real equal-rank Satake compactification. For any $\psi_{i-1}<\psi_{i}$ we may decompose

$$
\psi_{i}-\psi_{i-1}=\rho_{\mathbb{C} / \mathbb{R}}^{-1}(\alpha) \cup \eta \quad \text { for some } \alpha \in \mathbb{R} \Delta \text { and } \eta \subseteq \Delta_{\mathbb{C} / \mathbb{R}}^{0} .
$$

The subset $\eta$ is a union of components of $\Delta_{\mathbb{C} / \mathbb{R}}^{0}$ and

$$
\psi_{i-1} \cup\{\beta\} \text { is } \begin{cases}\text { connected } & \text { for } \beta \in \rho_{\mathbb{C} / \mathbb{R}}^{-1}(\alpha), \\ \text { disconnected } & \text { for } \beta \in \eta \text { if } i>1 .\end{cases}
$$

Proof. This follows from Definition 11 except for the assertion of "connected" in (3) as opposed to " $\delta$-connected"; for that use (the proof of) Corollary 16.

Proposition 18. Let $D_{\pi}^{*}$ be a real equal-rank Satake compactification. Then $\mathcal{B} \subset \mathcal{F}$.

Proof. By Lemma 14 it suffices to verify that any $\psi=\psi_{i} \in \mathcal{B}_{C}$ satisfies conditions (i) and (ii) from Definition 9. If $\psi_{i}^{+}-\psi_{i}$ had two elements modulo $\iota$, there would exist two incomparable elements $\psi_{i+1}$ and $\psi_{i+1}^{\prime}$ of $\mathcal{B}_{C}^{*}$ strictly containing $\psi_{i}$. For $C$ not type $F_{4}$, this is impossible by Lemma 14 and Proposition 10(i) since $\mathcal{B}_{C}^{*} \subseteq \widetilde{\mathcal{F}}_{C}^{*}$ is totally ordered. Type $F_{4}$ is excluded by Corollary 15 . Condition (i) follows.

As for condition (ii), let $\psi_{i+1} \in \mathcal{B}_{C}$ contain $\psi_{i}$; (i) implies that $\psi_{i+1} \supseteq \psi_{i}^{+}$. Then $\psi_{i+1}-\psi_{i}^{+}=\eta$ is a union of components of $\Delta_{\mathbb{C} / \mathbb{R}}^{0}$ by Lemma 17 ; these components belong to $\widetilde{\mathcal{F}}_{C}$ by Lemma 14.

Corollary 19. Let $D_{\pi}^{*}$ be a real equal-rank Satake compactification. Consider $\psi_{i-1}<\psi_{i} \in \mathcal{B}$ and let $\alpha \in \mathbb{R} \Delta$ be as in (2). If $\psi=\psi_{i-1}$ or if $\psi \in \mathcal{F}$ is a component of $\left(\psi_{i}-\psi_{i-1}\right) \cap \Delta_{\mathbb{C} / \mathbb{R}}^{0}$ (the noncompact case and the compact case respectively $)$, then $\psi^{+}-\psi=\rho_{\mathbb{C} / \mathbb{R}}^{-1}(\alpha)$.

Proof. In the noncompact case, (3) implies $\rho_{\mathbb{C} / \mathbb{R}}^{-1}(\alpha) \subseteq \psi^{+}-\psi$; the same inclusion holds in the compact case since $\psi_{i}$ is connected. However $\psi \in \mathcal{F}$ by the proposition (or by hypothesis in the compact case). Thus Definition 9(i) and Lemma 12 imply that $\psi^{+}-\psi$ is a single $c^{*}$-orbit. The corollary follows.

Theorem 20. Let $G$ be an almost $\mathbb{Q}$-simple semisimple group and let $D_{\pi}^{*}$ be a real equal-rank Satake compactification. If $G$ has an $\mathbb{R}$-simple factor $H$ with $\mathbb{R}$-rank $H=2$ and $\mathbb{C}$-type $B_{n}, C_{n}$, or $G_{2}$, assume that the Satake compactification associated to $\left.\pi\right|_{H}$ does not have a real boundary component of type $A_{1}$. Then $D_{\pi}^{*}$ is geometrically rational.

Remark 21. In particular, this implies Baily and Borel's result [3] on geometric rationality for the natural compactification of a Hermitian symmetric space except possibly when $G$ is the restriction of scalars of a group with $\mathbb{C}$-type $B_{n}$, $C_{n}$, or $G_{2}$. But the Hermitian condition excludes $G_{2}$ and implies for $B_{n}$ or $C_{n}$ that all simple factors of $G$ have the same $\mathbb{R}$-type, so $\delta$ is Galois invariant and Theorem 6 applies. 
Proof. For every component $C$ of $\mathbb{C} \Delta$, define

$$
\mathcal{F}_{C}^{\circ}=\mathcal{F}_{C}-\left\{\psi \in \mathcal{F}_{C} \mid \psi \text { is type } A_{1}\right. \text { and is covered by }
$$

$$
\left.C \text { which is type } B_{n}, C_{n} \text {, or } G_{2} \cdot\right\}
$$

and set $\mathcal{F}^{\circ}=\coprod_{C} \mathcal{F}_{C}^{\circ}$. If $\psi \in \mathcal{F}_{C}-\mathcal{F}_{C}^{\circ}$ belonged to $\mathcal{B}$, this would imply that $\mathbb{R}$-rank $C=2$. Since such a component is excluded by our hypotheses, Proposition 18 may be strengthened to $\mathcal{B} \subseteq \mathcal{F}^{\circ}$. Proposition 10(ii) implies that

(4) if $\psi \in \mathcal{B}_{C}$ and $\psi^{\prime} \in \mathcal{F}_{C}^{\circ}$ are incomparable, then $\psi \cup \psi^{\prime}$ is disconnected.

(If $C$ has type $F_{4}, \mathbb{R}$-rank $C=1$ by Corollary 15 which implies $\mathcal{B}_{C}=\{C\}$ and the above equation is vacuous.) It follows that

$$
\mathcal{B}_{C} \text { is totally ordered. }
$$

For if $\psi, \psi^{\prime} \in \mathcal{B}_{C}$ were incomparable, the union would be both disconnected by (4) and connected by Corollary 16.

Define

$$
\begin{aligned}
& \mathcal{K}_{n c}=\left\{\psi \mid \psi \text { is a component of } \kappa\left(\Delta_{\mathbb{C} / \mathbb{Q}}^{0}\right)\right\}, \\
& \mathcal{K}_{c}=\left\{\psi \in \mathcal{F}^{\circ} \mid \begin{array}{r}
\psi \text { is a component of }\left(\psi_{i}-\psi_{i-1}\right) \cap \Delta_{\mathbb{C} / \mathbb{R}}^{0} \text { for } \\
\text { some } \psi_{i-1}<\psi_{i} \in \mathcal{B} \text { with } \psi_{i} \nsubseteq \kappa\left(\Delta_{\mathbb{C} / \mathbb{Q}}^{0}\right)
\end{array}\right\},
\end{aligned}
$$

and set $\mathcal{K}=\mathcal{K}_{n c} \coprod \mathcal{K}_{c}$; we call the elements of $\mathcal{K}_{n c}$ noncompact and the elements of $\mathcal{K}_{c}$ compact. For a component $C$ of ${ }_{\mathbb{C}} \Delta$, we define $\mathcal{K}_{C}, \mathcal{K}_{C, n c}$, and $\mathcal{K}_{C, c}$ as usual.

Note that $\kappa\left(\Delta_{\mathbb{C} / \mathbb{Q}}^{0}\right)=\kappa\left(\varepsilon_{\mathbb{C} / \mathbb{R}}\left(\Delta_{\mathbb{R} / \mathbb{Q}}^{0}\right)\right)=\kappa\left(\varepsilon_{\mathbb{C} / \mathbb{R}}\left(\kappa\left(\Delta_{\mathbb{R} / \mathbb{Q}}^{0}\right)\right)\right) ;$ thus by Corollary 16

$$
\mathcal{K}_{n c}=\left\{\kappa\left(\Delta_{\mathbb{C} / \mathbb{Q}}^{0}\right) \cap C \mid \delta \cap \Delta_{\mathbb{C} / \mathbb{Q}}^{0} \cap C \neq \emptyset\right\} \subseteq \mathcal{B} \subseteq \mathcal{F}^{\circ} .
$$

We first show that if $\psi \in \mathcal{K}$ and $g \in \operatorname{Gal}(\mathbb{C} / \mathbb{Q})$, then

$$
g^{*} \psi \in \mathcal{F}^{\circ}, g^{*} \psi \subseteq \Delta_{\mathbb{C} / \mathbb{Q}}^{0} \text {, and } g^{*} \psi \text { is maximal among such sets. }
$$

It suffices to prove this for $g^{*}$ the identity since the definition of $\mathcal{F}^{\circ}$ depends only the $\mathbb{C}$-root system and since $\Delta_{\mathbb{C} / \mathbb{Q}}^{0}$ is $\operatorname{Gal}(\mathbb{C} / \mathbb{Q})$-invariant. Then the first assertion is part of the definition if $\psi$ is compact and follows from (6) if $\psi$ is noncompact. The second assertion, that $\psi \subseteq \Delta_{\mathbb{C} / \mathbb{Q}}^{0}$, is clear. For the final assertion, assume $\psi \in \mathcal{K}_{C}$ and suppose that there exists $\psi^{\prime} \in \mathcal{F}^{\circ}$ such that $\psi \subset \psi^{\prime} \subseteq \Delta_{\mathbb{C} / \mathbb{Q}}^{0} \cap C$. If $\psi$ is noncompact then it is $\delta$-connected and hence $\psi^{\prime}$ (being connected) must also be $\delta$-connected; this contradicts the fact that $\psi=\kappa\left(\Delta_{\mathbb{C} / \mathbb{Q}}^{0}\right) \cap C$ is the largest $\delta$-connected subset of $\Delta_{\mathbb{C} / \mathbb{Q}}^{0} \cap C$. If instead $\psi \subseteq \psi_{i}-\psi_{i-1}$ is compact, let $\alpha \in \mathbb{R}^{\Delta}$ be as in (2). Since $\psi^{+}-\psi=\rho_{\mathbb{C} / \mathbb{R}}^{-1}(\alpha)$ by Corollary 19, any connected set strictly containing $\psi$, such as $\psi^{\prime}$, must contain an element of $\rho_{\mathbb{C} / \mathbb{R}}^{-1}(\alpha)$. Thus (a) $\psi_{i}-\psi_{i-1} \subseteq \Delta_{\mathbb{C} / \mathbb{Q}}^{0}$ by (2) and (b) $\psi_{i-1} \cup \psi^{\prime}$ is connected by (3). If $i=1$, (a) implies $\psi_{1} \subseteq \kappa\left(\Delta_{\mathbb{C} / \mathbb{Q}}^{0}\right)$ which contradicts the 
definition of $\mathcal{K}_{c}$. If $i>1$, the same argument shows that $\psi_{i-1} \nsubseteq \psi^{\prime}$ and hence $\psi_{i-1}$ and $\psi^{\prime}$ are incomparable; this contradicts (4) and (b).

We now prove that

$$
\mathcal{K} \text { is } \operatorname{Gal}(\mathbb{C} / \mathbb{Q}) \text {-invariant, }
$$

that is, given $\psi \in \mathcal{K}_{C}$ and $g \in \operatorname{Gal}(\mathbb{C} / \mathbb{Q})$ we will show that $g^{*} \psi \in \mathcal{K}$. Since we can assume $G$ is not $\mathbb{Q}$-anisotropic, $g^{*} \psi \neq g^{*} C$. Let $i$ be minimal such that $g^{*} \psi<\psi_{i}$ for some $\psi_{i} \in \mathcal{B}$ and consider some $\psi_{i-1}<\psi_{i}$. We know that $\psi_{i} \nsubseteq £\left(\Delta_{\mathbb{C} / \mathbb{Q}}^{0}\right)$, else the maximality in $(7)$ would be contradicted. There are three cases.

Case 1: $i=1$. Let $\alpha$ and $\eta$ be as in Lemma 17. We have $\rho_{\mathbb{C} / \mathbb{R}}^{-1}(\alpha) \cap \Delta_{\mathbb{C} / \mathbb{Q}}^{0}=\emptyset$ else $\psi_{1} \subseteq \kappa\left(\Delta_{\mathbb{C} / \mathbb{Q}}^{0}\right)$. Thus $g^{*} \psi \in \mathcal{F}^{\circ}$ is contained in a component of $\eta=\psi_{1} \cap \Delta_{\mathbb{C} / \mathbb{R}}^{0}$; since Proposition 10 and Lemmas 14 and 17 imply such a component is in $\mathcal{F}^{\circ}$, and hence $\mathcal{K}_{c}$, the maximality in (7) implies $g^{*} \psi$ equals this component.

Case 2: $i>1$ and $\psi_{i-1} \leq g^{*} \psi$. In this case, $\psi_{i-1} \subseteq g^{*} \psi \subseteq \Delta_{\mathbb{C} / \mathbb{Q}}^{0}$ where $\psi_{i-1}$ is nonempty and $\delta$-connected and $g^{*} \psi$ is connected. This implies $g^{*} \psi$ is $\delta$-connected and hence equals a component of $\kappa\left(\Delta_{\mathbb{C} / \mathbb{Q}}^{0}\right)$ by the maximality in (7). So $g^{*} \psi \in \mathcal{K}_{n c}$.

Case 3: $i>1$ and $\psi_{i-1} \not \leq g^{*} \psi$. Since $\psi_{i-1} \cup g^{*} \psi$ is disconnected by $(4), g^{*} \psi$ is contained in a connected component of $\left(\psi_{i}-\psi_{i-1}\right) \cap \Delta_{\mathbb{C} / \mathbb{R}}^{0}$ by (3); as in Case 1 , this component must be in $\mathcal{K}_{c}$ and $g^{*} \psi$ equals it.

This finishes the proof that $\mathcal{K}$ is $\operatorname{Gal}(\mathbb{C} / \mathbb{Q})$-invariant.

Let $\widehat{\mathcal{K}}=\operatorname{Gal}(\mathbb{C} / \mathbb{Q}) \cdot \mathcal{K}_{n c}$. We claim that if $\psi \in \widehat{\mathcal{K}}_{C}$ is compact, say $\psi \subseteq$ $\left(\psi_{i}-\psi_{i-1}\right) \cap \Delta_{\mathbb{C} / \mathbb{R}}^{0}$, then $\psi_{i-1}=\kappa\left(\Delta_{\mathbb{C} / \mathbb{Q}}^{0}\right) \cap C$. In the case $i=1$, the claim asserts that $\kappa\left(\Delta_{\mathbb{C} / \mathbb{Q}}^{0}\right) \cap C=\emptyset$; this holds since otherwise $\kappa\left(\Delta_{\mathbb{C} / \mathbb{Q}}^{0}\right) \cap C \supseteq \psi_{1}$ (by (5)) which contradicts the definition of $\mathcal{K}_{c}$. As for the case $i>1$, let $g \in \operatorname{Gal}(\mathbb{C} / \mathbb{Q})$ be such that $g^{*} \psi$ is noncompact. Then $g^{*} \psi \in \mathcal{B}$ and $g^{*} \psi_{i-1}$ are incomparable; let $\tilde{\psi} \in \mathcal{B}$ contain $g^{*} \psi$ and be maximal such that $\tilde{\psi}$ and $g^{*} \psi_{i-1}$ are incomparable. It follows from (4) and Lemma 17 that $g^{*} \psi_{i-1} \subseteq \Delta_{\mathbb{C} / \mathbb{R}}^{0}$ and hence $\psi_{i-1} \subseteq \Delta_{\mathbb{C} / \mathbb{Q}}^{0}$. Thus $\psi_{i-1} \subseteq \kappa\left(\Delta_{\mathbb{C} / \mathbb{Q}}^{0}\right) \cap C \in \mathcal{B}_{C}$, while $\psi_{i} \nsubseteq £\left(\Delta_{\mathbb{C} / \mathbb{Q}}^{0}\right)$. The claim now follows from (5).

Assume now that $\widehat{\mathcal{K}}_{C} \neq \emptyset$ for one and hence all components $C$. For a fixed $C$, it follows from the above claim and Corollary 19 that $\psi^{+}-\psi$ is independent of the choice of $\psi \in \widehat{\mathcal{K}}_{C}$ and, if there does not exist a noncompact element in $\widehat{\mathcal{K}}_{C}$, then $\delta \cap C=\psi^{+}-\psi$. It follows that

(9) $\beta \in \omega\left(\Delta_{\mathbb{C} / \mathbb{Q}}^{0}\right) \cap C \quad \Longleftrightarrow \quad \beta \notin \psi^{+}-\psi$ for some (and hence any) $\psi \in \widehat{\mathcal{K}}_{C}$.

For in the case that there exists $\psi \in \widehat{\mathcal{K}}_{C}$ noncompact, equation (9) holds since $\psi=\kappa\left(\Delta_{\mathbb{C} / \mathbb{Q}}^{0}\right) \cap C$, while in the case that every $\psi \in \widehat{\mathcal{K}}_{C}$ is compact, $\omega\left(\Delta_{\mathbb{C} / \mathbb{Q}}^{0}\right) \cap C$ is simply the complement of $\delta \cap C=\psi^{+}-\psi$ in $C$. Since the right hand side of (9) is $\operatorname{Gal}(\mathbb{C} / \mathbb{Q})$-invariant, condition (i) of Theorem 5 holds. Also if $g \in \operatorname{Gal}(\mathbb{C} / \mathbb{Q})$ and 
$\psi \in \mathcal{K}$ is a component of $\kappa\left(\Delta_{\mathbb{C} / \mathbb{Q}}^{0}\right)$, then $g^{*} \psi \in \mathcal{K}$ and thus either $g^{*} \psi \subseteq \kappa\left(\Delta_{\mathbb{C} / \mathbb{Q}}^{0}\right)$ or $g^{*} \psi \subseteq \Delta_{\mathbb{C} / \mathbb{R}}^{0} ;$ this verifies condition (ii) of Theorem 5 .

The remaining case is where $\widehat{\mathcal{K}}_{C}=\emptyset$ for all $C$. Then $\bigcup_{g \in \operatorname{Gal}(\mathbb{C} / \mathbb{Q})} g^{*} \mathcal{B} \cap C$ is totally ordered for any $C$; this may be proved similarly to (8) but more easily. Let $\psi_{1} \in \mathcal{B}_{C}$ and compare $g^{*} \psi_{1}$ with $\psi_{1}^{\prime} \in \mathcal{B}_{g^{*} C}$. Say $g^{*} \psi_{1} \leq \psi_{1}^{\prime}$. We must have $g^{*}(\delta \cap C) \subseteq \delta \cap g^{*} C$, since otherwise $\kappa\left(\Delta_{\mathbb{C} / \mathbb{Q}}^{0}\right)$ would not be empty. Since $g^{*}$ commutes with $c^{*}$ (Corollary 13) and $\delta \cap C$ has only one element modulo $c^{*}$ (by equation (5)), we have equality. Thus $\delta$ is Galois invariant and the theorem follows from Theorem 6 .

\section{Exceptional cases}

It remains to handle the cases excluded from Theorem 20. In contrast to the situation of that theorem, where geometric rationality was automatic with no rationality assumption on $(\pi, V)$, these exceptional cases are only geometrically rational under a certain condition which depends on the $\mathbb{Q}$-rank.

Theorem 22. Let $G$ be an almost $\mathbb{Q}$-simple semisimple group which is the restriction of scalars of a group $G^{\prime}$ with $\mathbb{C}$-type $B_{n}, C_{n}$, or $G_{2}$. Let $D_{\pi}^{*}$ be a real equal-rank Satake compactification. Assume $G$ has an $\mathbb{R}$-simple factor $H$ with $\mathbb{R}$-rank $H=2$ for which the Satake compactification associated to $\left.\pi\right|_{H}$ has a real boundary component of type $A_{1}$.

(i) In the case $\mathbb{Q}$-rank $G=2, D_{\pi}^{*}$ is geometrically rational if and only if $\delta$ is Galois invariant.

(ii) In the case $\mathbb{Q}$-rank $G=1, D_{\pi}^{*}$ is geometrically rational if and only if $\delta \cap \Delta_{\mathbb{C} / \mathbb{Q}}^{0}$ is empty or meets every component of $\mathbb{C} \Delta$ with $\mathbb{R}$-rank $\geq 2$.

Proof. Let $C$ be the component of $\mathbb{C}_{\mathbb{C}} \Delta$ corresponding to $H$ and let $\alpha_{1} \in \delta \cap C$ correspond to the real boundary component of type $A_{1}$. By Lemma 17 and our hypotheses, $\alpha_{1}$ is at an end of $C$ and (if we denote its unique neighbor by $\alpha_{2}$ ) we have $C-\left\{\alpha_{1}, \alpha_{2}\right\}=\Delta_{\mathbb{C} / \mathbb{R}}^{0} \cap C \subseteq \Delta_{\mathbb{C} / \mathbb{Q}}^{0} \cap C$. If $H$ has $\mathbb{C}$-type $C_{n}$, the classification of semisimple Lie algebras over $\mathbb{R}[1],[13$, Chapter $\mathrm{X}$, Table VI] shows that the two adjacent non- $\mathbb{R}$-anisotropic roots imply $H$ is $\mathbb{R}$-split; thus $C_{n}$ only occurs for $n=2$ and we can absorb this case into that of $B_{n}$. Classification theory over $\mathbb{R}$ also shows that if $n>2$, the simple root $\alpha_{1}$ is long.

For (i) we only need to prove that $\delta$ is Galois invariant under the assumption that (i) and (ii) of Theorem 5 hold; the opposite direction was already proved in Theorem 6. Thus assume that $\mathbb{Q}$-rank $G=2$. Then for all $g \in \operatorname{Gal}(\mathbb{C} / \mathbb{Q})$ we have $\kappa\left(\Delta_{\mathbb{C} / \mathbb{Q}}^{0}\right) \cap g^{*} C \subseteq \Delta_{\mathbb{C} / \mathbb{Q}}^{0} \cap g^{*} C=g^{*}\left(C-\left\{\alpha_{1}, \alpha_{2}\right\}\right) \subseteq \omega\left(\Delta_{\mathbb{C} / \mathbb{Q}}^{0}\right) \cap g^{*} C$. Since $g^{*} \alpha_{1}$ is orthogonal to $g^{*}\left(C-\left\{\alpha_{1}, \alpha_{2}\right\}\right)$, it follows that $g^{*} \alpha_{1} \notin \omega\left(\Delta_{\mathbb{C} / \mathbb{Q}}^{0}\right) \cap g^{*} C$ if and only if $g^{*} \alpha_{1} \in \delta \cap g^{*} C$. But the first condition is independent of $g$ by condition (i) of Theorem 5 while the second condition holds for $g=e$, so we must have $g^{*} \alpha_{1} \in \delta \cap g^{*} C$ for all $g \in \operatorname{Gal}(\mathbb{C} / \mathbb{Q})$. If $\#\left(\delta \cap g_{0}^{*} C\right)>1$ for some $g_{0} \in \operatorname{Gal}(\mathbb{C} / \mathbb{Q})$ then $\mathbb{R}$-rank $g_{0}^{*} C=2$ by Propositions 10 (ii) and 18 , in which case $g_{0}^{*}\left(C-\left\{\alpha_{1}, \alpha_{2}\right\}\right)=\Delta_{\mathbb{C} / \mathbb{R}}^{0} \cap g_{0}^{*} C$; thus the additional element of $\delta \cap g_{0}^{*} C$ 
must be $g_{0}^{*} \alpha_{2}$. In this case however $\kappa\left(\Delta_{\mathbb{C} / \mathbb{Q}}^{0}\right)=\emptyset$ so that for all $g \in \operatorname{Gal}(\mathbb{C} / \mathbb{Q})$, $g^{*} \alpha_{2} \notin \omega\left(\Delta_{\mathbb{C} / \mathbb{Q}}^{0}\right) \cap g^{*} C$ if and only if $g^{*} \alpha_{2} \in \delta \cap g^{*} C$. Another application of condition (i) from Theorem 5 concludes the proof that $\delta$ is Galois invariant in this case.

For (ii) assume that $\mathbb{Q}$-rank $G=1$. Write $G=R_{k / \mathbb{Q}} G^{\prime}$ where $k$ is a finite extension of $\mathbb{Q}$ (totally real by Lemma 14 ) and $G^{\prime}$ is an almost $k$-simple group with $k$-rank $=1$ and $\mathbb{C}$-type $B_{n}$ or $G_{2}$. The classification of semisimple groups over $\mathbb{Q}[24]$ shows the case $G_{2}$ cannot occur and that in the case $B_{n}$ the unique simple root which is not $k$-anisotropic is the long root at the end of the Dynkin diagram. Since $\Delta_{\mathbb{C} / \mathbb{Q}}^{0} \cap C$ is thus connected for every component $C$ of $\mathbb{C} \Delta$, it follows that

$$
\kappa\left(\Delta_{\mathbb{C} / \mathbb{Q}}^{0}\right) \cap C \text { is nonempty } \quad \Longleftrightarrow \quad \delta \cap \Delta_{\mathbb{C} / \mathbb{Q}}^{0} \text { meets } C .
$$

If this condition holds, then $\kappa\left(\Delta_{\mathbb{C} / \mathbb{Q}}^{0}\right) \cap C=\Delta_{\mathbb{C} / \mathbb{Q}}^{0} \cap C$, while if the condition does not hold, then $\delta \cap C=C-\left(\Delta_{\mathbb{C} / \mathbb{Q}}^{0} \cap C\right)$. In either case, $\omega\left(\Delta_{\mathbb{C} / \mathbb{Q}}^{0}\right) \cap C=\Delta_{\mathbb{C} / \mathbb{Q}}^{0} \cap C$. Furthermore

$$
\Delta_{\mathbb{C} / \mathbb{Q}}^{0} \cap C \nsubseteq \Delta_{\mathbb{C} / \mathbb{R}}^{0} \quad \Longleftrightarrow \quad \mathbb{R}-\operatorname{rank} C \geq 2 .
$$

Part (ii) now easily follows from Theorem 5.

Remarks 23. Assume $G$ satisfies the hypotheses of Theorem 22 .

(i) In the $\mathbb{Q}$-rank 2 case, if $D_{\pi}^{*}$ is geometrically rational then all $\mathbb{R}$-simple factors of $G$ have $\mathbb{R}$-rank $=2$. For the proof shows that every component $g^{*} C$ has an $A_{1}$ boundary component corresponding to $g^{*} \alpha_{1} \in \delta \cap g^{*} C$ and that if $n>2$ then $\alpha_{1}$ is long. However if $\mathbb{R}$-rank $g^{*} C>2$ then the entry for $B_{n}$ in Figure 1 implies that $\delta \cap g^{*} C$ is a singleton short root.

(ii) In the $\mathbb{Q}$-rank 1 case, $\delta \cap \Delta_{\mathbb{C} / \mathbb{Q}}^{0}$ automatically meets any component with $\mathbb{R}$-rank $>2$; this follows from the proof and the entry for $B_{n}$ in Figure 1. Thus if there is any component with $\mathbb{R}$-rank $>2$, the condition for geometric rationality is that $\delta \cap \Delta_{\mathbb{C} / \mathbb{Q}}^{0}$ meets every component with $\mathbb{R}-$ rank $=2$.

(iii) Also in the $\mathbb{Q}$-rank 1 case, if $\mathbb{C}$-rank $G^{\prime}>2$ then $G$ has a component with $\mathbb{R}$-rank 1 . For by the proof above, $G^{\prime}$ (up to strict $k$-isogeny) is the special orthogonal group of a quadratic form $q$ in at least 7 variables with $k$-index 1 . Thus we can decompose $q$ into a hyperbolic plane and a $k$-anisotropic form $q^{\prime}$ in at least 5 variables. Since every quadratic form in at least 5 variables is isotropic at every non-archimedean place [15, VI.2.12], $q^{\prime}$ must be anisotropic at some real place by the HasseMinkowski principle [15, VI.3.5].

Examples 24. Let $k$ be a real quadratic extension of $\mathbb{Q}$. In the Satake diagrams below, the roots in $\Delta_{\mathbb{C} / \mathbb{R}}^{0}$ are colored black, while roots in $\Delta_{\mathbb{C} / \mathbb{Q}}^{0}$ are enclosed in a dotted box. Nodes placed vertically above each other form a $\operatorname{Gal}(\mathbb{C} / \mathbb{Q})$-orbit. The elements of $\delta$ are so labeled. 
(i) Let $q$ be a $k$-anisotropic form in three variables, $\mathbb{R}$-anisotropic at one real place and of signature $(2,1)$ at the other. Let $G^{\prime}$ be the orthogonal group of $h \oplus h \oplus q$, where $h$ is hyperbolic space, and let $G=R_{k / \mathbb{Q}} G^{\prime}$. Then both of the Satake compactifications
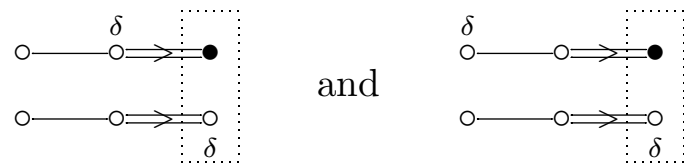

are real equal-rank. The first is geometrically rational (Theorem 20 applies) and the second is not (Theorem 22(i) applies). More specifically, condition (i) from Theorem 5 fails in the second example; this example also illustrates Remark 23(i).

If $q$ is instead nonsingular of dimension 1 , then of three real equalrank Satake compactifications

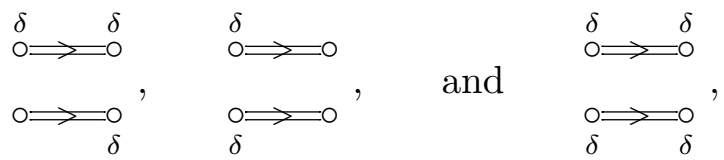

Theorem 22(i) shows that the first is not geometrically rational and the last two are. Similar examples can be constructed with $q$ totally $\mathbb{R}$-anisotropic in any odd number of variables or where $G^{\prime}$ is the split form of $G_{2}$.

(ii) Let $G^{\prime}$ be the orthogonal group of $h \oplus q$, where $q$ is a $k$-anisotropic form in three variables which has signature $(2,1)$ at both real places, and let $G=R_{k / \mathbb{Q}} G^{\prime}$. Among the real equal-rank Satake compactifications
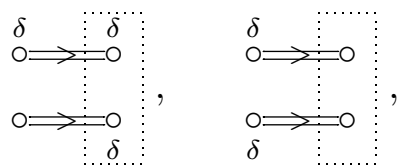

and

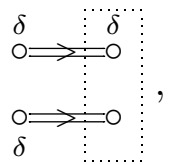

the first two are geometrically rational (even though in the first $\delta$ is not Galois invariant) and the last is not; this illustrates Theorem 22(ii).

(iii) Let $G^{\prime}$ be the orthogonal group of $h \oplus q$, where $q$ is a $k$-anisotropic form in five variables which has signature $(4,1)$ at one real place and is $\mathbb{R}$-anisotropic at the other (see Remark 23(iii)) and let $G=R_{k / \mathbb{Q}} G^{\prime}$. There are two real equal-rank Satake compactifications covered by Theorem 22(ii),

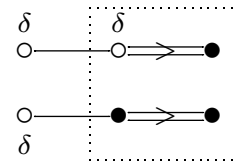

and

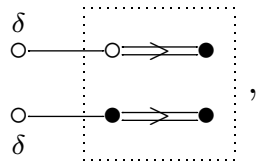

both of which are geometrically rational. If, however, $k$ is a totally real degree 3 extension of $\mathbb{Q}$ and $q$ has signature $(4,1),(5,0)$, and $(3,2)$ at the three real places, the two real equal-rank Satake compactifications 
covered by Theorem 22(ii) are
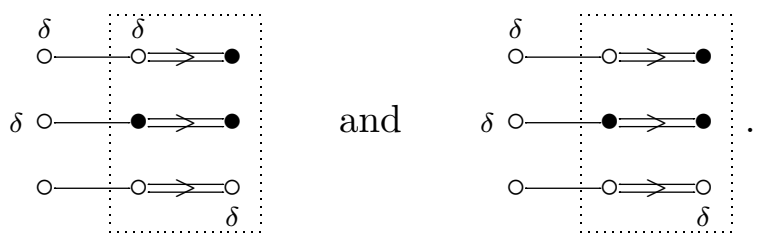

Here the first is geometrically rational and the second is not; this illustrates Remark 23(ii).

\section{References}

[1] S. Araki, On root systems and an infinitesimal classification of irreducible symmetric spaces, J. Math. Osaka City Univ. 13 (1962), 1-34.

[2] A. Ash, D. Mumford, M. Rapoport, and Y. Tai, Smooth compactification of locally symmetric varieties, Math Sci Press, Brookline, 1975.

[3] W. Baily and A. Borel, Compactification of arithmetic quotients of bounded symmetric domains, Ann. of Math. 84 (1966), 442-528.

[4] A. Borel, Ensembles fondamentaux pour les groupes arithmétiques, Colloque sur la Théorie des Groupes Algébriques, Tenu à Bruxelles du 5 au 7 juin 1962, Centre Belge de Recherches Mathématiques, 1962, 23-40.

[5] _ March 19, 2002, letter to L. Saper.

[6] A. Borel and W. Casselman, $L^{2}$-cohomology of locally symmetric manifolds of finite volume, Duke Math. J. 50 (1983), 625-647.

[7] A. Borel and J. Tits, Groupes réductifs, I.H.E.S. Publ. Math. 27 (1965), 55-151.

[8] W. Casselman, Geometric rationality of Satake compactifications, Algebraic groups and Lie groups, Austral. Math. Soc. Lect. Ser., 9, Cambridge Univ. Press, Cambridge, 1997, 81-103.

[9] M. Goresky, G. Harder, and R. MacPherson, Weighted cohomology, Invent. Math. 116 (1994), 139-213.

[10] M. Goresky and R. MacPherson, Weighted cohomology of Satake compactifications, Centre de recherches mathématiques, preprint \#1593, 1988.

[11] Harish-Chandra, Spherical functions on a semisimple Lie group. I, Amer. J. Math. 80 (1958), 241-310.

[12] S. Helgason, A duality for symmetric spaces with applications to group representations, Adv. Math. 5 (1970), 1-154.

[13] _ Differential geometry, Lie groups, and symmetric spaces, Pure and Applied Mathematics, 80, Academic Press, New York, 1978.

[14] A. Knapp, Lie groups, beyond an introduction, Birkhäuser, Boston, 1996.

[15] T. Y. Lam, The algebraic theorem of quadratic forms, Benjamin, Reading, Mass., 1973.

[16] Y. Matsushima and S. Murakami, On vector bundle valued harmonic forms and automorphic forms on symmetric Riemannian manifolds, Ann. of Math. 78 (1963), 365-416.

[17] C. Moore, Compactifications of symmetric spaces II: The Cartan domains, Amer. J. Math. 86 (1964), 358-378.

[18] M. Rapoport, 1986, letter to A. Borel.

[19] On the shape of the contribution of a fixed point on the boundary: The case of $\mathbb{Q}$-rank 1 (with an appendix by L. Saper and M. Stern), The Zeta functions of Picard modular surfaces, Univ. Montréal, Montréal, QC, 1992, 479-488.

[20] L. Saper, L-modules and micro-support, preprint, math.RT/0112251, 2001.

[21] I. Satake, On representations and compactifications of symmetric Riemannian spaces, Ann. of Math. 71 (1960), 77-110. 
[22] _ On compactifications of the quotient spaces for arithmetically defined discontinuous groups, Ann. of Math. 72 (1960), 555-580.

[23] R. P. Stanley, Enumerative combinatorics, 1, Cambridge Univ. Press, Cambridge, 1997.

[24] J. Tits, Classification of algebraic semisimple groups, Algebraic Groups and Discontinuous Subgroups, Proc. Sympos. Pure Math., 9, Amer. Math. Soc., Providence, 1966, 33-62.

[25] S. Zucker, Satake compactifications, Comment. Math. Helv. 58 (1983), 312-343.

[26] _ L L L -cohomology and intersection homology of locally symmetric varieties, II, Compositio Math. 59 (1986), 339-398.

Department of Mathematics, Duke University, Box 90320, Durham, NC 27708, U.S.A.

E-mail address: saper@math.duke.edu

$U R L:$ http://www.math.duke.edu/faculty/saper 